\title{
Phase Separation Behavior and System Properties of Aqueous Two-Phase Systems with Polyethylene Glycol and Different Salts: Experiment and Correlation
}

\author{
Haihua Yuan, ${ }^{1}$ Yang Liu, ${ }^{1}$ Wanqian Wei, ${ }^{1}$ and Yongjie Zhao ${ }^{2}$ \\ ${ }^{1}$ Department of Biology and Guangdong Provincial Key Laboratory of Marine Biotechnology, Shantou University, Shantou, \\ Guangdong 515063, China \\ ${ }^{2}$ Department of Mechanical Engineering, College of Engineering, Shantou University, Shantou, Guangdong 515063, China
}

Correspondence should be addressed to Yang Liu; liuyanglft@stu.edu.cn

Received 15 October 2014; Revised 22 January 2015; Accepted 3 February 2015

Academic Editor: Robert M. Kerr

Copyright (C) 2015 Haihua Yuan et al. This is an open access article distributed under the Creative Commons Attribution License, which permits unrestricted use, distribution, and reproduction in any medium, provided the original work is properly cited.

\begin{abstract}
The phase separation behaviors of PEG1000/sodium citrate, PEG4000/sodium citrate, PEG1000/ammonium sulfate, and PEG4000/ammonium sulfate aqueous two-phase systems were investigated, respectively. There are two distinct situations for the phase separation rate in the investigated aqueous two-phase systems: one state is top-continuous phase with slow phase separation rate and strong bottom-continuous phase with fast phase separation rate and weak volume ratio dependence. The system properties such as density, viscosity, and interfacial tension between top and bottom phases which have effects on the phase separation rate of aqueous two-phase systems were measured. The property parameter differences between the two phases increased with increasing tie line length and then improved the phase separation rate. Moreover, a modified correlation equation including the phase separation rate, tie line length, and physical properties of the four aqueous two-phase systems has been proposed and successfully tested in the bottom-continuous phase, whose coefficients were estimated through regression analysis. The predicted results of PEG1000/sodium citrate aqueous two-phase systems were verified through the stationary phase retention in the cross-axis countercurrent chromatography.
\end{abstract}

\section{Introduction}

In the 1960s, aqueous two-phase systems (ATPS) have been exploited to process different biological sources for the recovery of biological products, such as amino acids, proteins, nucleic acids, antibodies, cells, and organelles [1-5]. ATPS have also become an attractive separation technology due to the mild conditions, the low cost of the phase forming materials, the simplicity of the process, and the easy scale-up to an industrial level. Recently, polymer/salt ATPS, especially polyethylene glycol (PEG)/salt ATPS, have been extensively applied in the separation of many biological products [6-10]. PEG/salt ATPS have advantages of low cost, low viscosity of both the phases, and rapid phase separation rate, so this kind of ATPS has been used for the production of biological molecules on an industrial scale. For example, the scale-up isolation of formate dehydrogenase from $30-50 \mathrm{Kg}$ of wet cells of Candida boidinii was investigated by Kroner et al. [11]. The separation of engineered protein was also successfully scaled up to $1200 \mathrm{~L}$ by Selber et al. [12]. It displays that ATPS have potential for the large-scale implementation in biotechnology.

Multistage extraction process such as high-speed countercurrent chromatography (HSCCC) has been applied further to improve the target product purity compared with the single-stage extraction. Organic/aqueous solutions system has been widely used in HSCCC due to the short phase separation time $(<30 \mathrm{~s})$ [13], while ATPS application in HSCCC is limited due to the long phase separation time $(>50 \mathrm{~s})$. The phase separation time of ATPS increases to 3-5 min with the increasing components concentrations [14,15]. Moreover, the emulsion phenomenon in ATPS is usually serious due to the long phase separation time in the continuous process, which makes it difficult to separate the target products thoroughly. 
Many works focused on HSCCC instrument design for ATPS separation to eliminate the emulsion [16-18]. There are few reports about the phase separation rate and physical properties of ATPS $[14,15]$ for HSCCC.

Much of the research $[19,20]$ in ATPS has found that polyethylene glycol/organic salts ATPS could be employed as a viable and potentially useful tool for separating proteins instead of the conventional PEG/inorganic systems. The main advantages of PEG/sodium citrate ATPS are the biodegradability and nontoxicity of the organic anion when comparing with the high eutrophication potential of inorganic ions [21] and its harmful impact on the environment. In our present work, the physical properties and phase separation behaviors of the ATPS including PEG/organic salts (PEG/sodium citrate) and PEG/inorganic salts (PEG/ammonium sulfate) systems were investigated for comparisons. Here, ammonium sulfate was selected as the inorganic salt due to its frequent use in protein precipitation. The phase separation rate and the physical properties of ATPS such as density, viscosity, and interfacial tension were measured and correlated with the tie line length (TLL) and ATPS $V_{r}$ which can contribute to the HSCCC application using ATPS for protein separation. Finally, the stationary phase retention of PEG/sodium citrate ATPS in the cross-axis countercurrent chromatography ( $X$ axis CCC) was investigated based on the above correlated models, in which the $X$-axis CCC was designed and fabricated in our laboratory.

\section{Materials and Methods}

2.1. Chemicals. PEG1000 and PEG4000 (GR, $\geq 95 \%$ mass purity) were purchased from Merck (Shanghai, China). Sodium citrate (GR, $\geq 99.5 \%$ mass purity) and ammonium sulfate (GR, $\geq 99.5 \%$ mass purity) were of analytical grade from local sources. Aqueous solutions were prepared with deionized and doubly distilled water.

2.2. Preparation of ATPS. ATPS were prepared from stock solutions of PEG $(50 \% \mathrm{w} / \mathrm{w})$, sodium citrate $(30 \% \mathrm{w} / \mathrm{w})$, and ammonium sulfate $(40 \% \mathrm{w} / \mathrm{w})$. Phase boundaries of all ATPS were obtained by cloud-point measurements in a thermostatic bath (DC-0506, Shanghai Jingtian Electronic Instrument Co. Ltd) of $25^{\circ} \mathrm{C}$. The temperature was maintained with an uncertainty of $\pm 0.05^{\circ} \mathrm{C}$. The PEG/salt ATPS with five tie lines lengths $(30,35,40,45$, and 50$)$ were equilibrated for $12 \mathrm{~h}$ at $25 \pm 0.05^{\circ} \mathrm{C}$ before use. The compositions of the tie lines were determined by the $V_{r}$ and the lever rule [22]. The $V_{r}$ was determined in graduated centrifuge tubes with an uncertainty of $\pm 0.1 \mathrm{~mL}$.

2.3. Density Measurements. The top and bottom phase densities were measured using a $10 \mathrm{~mL}$ density bottle with an uncertainty of $\pm 0.1 \mathrm{~kg} \cdot \mathrm{m}^{-3}$ at $25 \pm 0.05^{\circ} \mathrm{C}$. The measurements were done in triplicate, and the average value was reported.

2.4. Viscosity Measurements. The viscosity of all ATPS was measured using digital rotary viscosimeter (NDJ-5S, Shanghai Jingtian Electronic Instrument Co. Ltd) with a precision of $\pm 0.0001 \mathrm{~Pa} \cdot \mathrm{s}$. The samples were first maintained at working temperature in thermostatic bath with an uncertainty of $\pm 0.05^{\circ} \mathrm{C}$ for $10 \mathrm{~min}$, and measurements were done in triplicate.

2.5. Interfacial Tension Measurements. The interfacial tension was measured by a drop volume method $[23,24]$ at $25^{\circ} \mathrm{C}$. Some burettes having capillaries with different outer diameters were used, since the interfacial tension changed with the composition of ATPS. Interfacial tension between the two phases was determined by the numbers and volumes of fallen drops from the top phase solution and the density difference between the top and bottom phases. The interfacial tension is then given by

$$
\begin{gathered}
\gamma=\frac{\Delta \rho V g}{2 \pi r \psi}, \\
\psi=0.9045-0.7294\left(\frac{r}{V^{1 / 3}}\right)+0.4293\left(\frac{r}{V^{1 / 3}}\right)^{2}, \\
\left(0.3<\frac{r}{V^{1 / 3}}<1.2\right), \\
\psi=1.007-1.479\left(\frac{r}{V^{1 / 3}}\right)+1.829\left(\frac{r}{V^{1 / 3}}\right)^{2}, \\
\left(\frac{r}{V^{1 / 3}} \leq 0.3\right),
\end{gathered}
$$

where $\gamma$ is the interfacial tension between the phases of ATPS, $\Delta \rho$ is the density difference of the two phases, $V$ is the average volume of drops, $r$ is the radius of the tip of burette, $g$ is the local value for the acceleration due to gravity, and $\psi$ is the correction factors determined by the values of $V$ and $r$. The uncertainty of interfacial tension measurement was $\pm 0.0001 \mathrm{mN} \cdot \mathrm{m}^{-1}$. All the measurements were done in triplicate.

2.6. Phase Separation Rate. ATPS mixing was performed in centrifuge tubes with $10 \mathrm{~mL}$ volume. The time of mixing was 10 min by manual operation. For all the experiments, the time for phase separation was defined as the time needed for most bulk of the phases to separate and a horizontal interface was formed. Some small drops of one phase which remained in the other phase were ignored. The measurements were done in triplicate with a precision of $\pm 0.1 \mathrm{~s}$.

Kaul et al. found that the kinetic behavior depends greatly on which of the phases is continuous and that the properties of the continuous phase strongly influence the movement of the drops of the dispersed phase [25-27]. Two typical batches' separation is shown in Figure 1. For the top-continuous ATPS, the drops of bottom phase descend in centrifuge tubes and form a settling front; for the bottom-continuous ATPS, the drops of top phase ascend in centrifuge tubes and form a rising front. It can be seen that the process of coalescence is slower than droplet descent and ascent due to the existence of the front.

The problems of phase continuity and phase inversion are important for the phase separation behavior and phase 


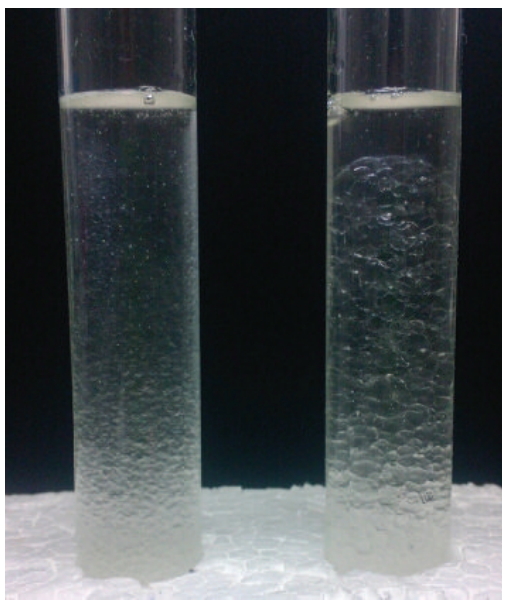

Figure 1: Two typical batch separations for PEG4000/ammonium sulfate ATPS. Left: top-continuous phase; right: bottom-continuous phase.

separation rate. It has been shown that the phase continuity and phase inversion occurred as the $V_{r}$ changed according to Kaul et al's methods [28]. During the ATPS phase separation process, when the descending droplets in the dispersive region were observed, it means a top-continuous phase, while ascending droplets means a bottom-continuous phase. For the same TLL of ATPS, different composition concentrations with various $V_{r}$ make different continuous phase. Hence, both the top-continuous region and bottom-continuous region can be seen in the same phase diagram. A locus of phase inversion points is found in the phase diagram between topcontinuous region and bottom-continuous region, creating a phase inversion band. To determine this band, four ATPS with five different TLL were chosen, and the separation behavior of the ATPS at TLL with different $V_{r}$ was investigated.

An equation can express the correlation between the phase separation rates and the physical properties of PEG/salt ATPS. The comprehensive efficiency of the physical properties including density, viscosity, and interface tension can be represented by Morton number $\left(M_{0}\right)$ [29-31]. Hence, a generalized correlation equation given by Mistry and Golob $[4,27]$ was developed for the ATPS with different PEG molar masses and salt relating the Morton number $\left(M_{0}\right)$, the ratio of interfacial tension to surface tension, $V_{r}$, and TLL:

$$
\begin{gathered}
\frac{d h}{d t}\left(\frac{\mu_{c}}{\lambda}\right)=a M_{0}^{b}\left(\frac{\gamma}{\sigma_{c}}\right)^{c}\left(\frac{V_{\mathrm{tp}}}{V_{\mathrm{bp}}}\right)^{d}(\mathrm{TLL})^{e}, \\
M_{0}=\frac{\Delta \mu^{4} g}{\gamma^{3} \Delta \rho},
\end{gathered}
$$

where $a, b, c, d, e$ are the coefficients of the equation, $d h / d t$ is the phase separation rate, $\mu_{c}$ is the viscosity of the continuous phase, $\sigma_{c}$ is the surface tension of continuous phase, $V_{\mathrm{tp}}$ is the volume of top phase, $V_{\mathrm{bp}}$ is the volume of bottom phase, and $\Delta \mu$ is the viscosity difference between top and bottom phases.
2.7. Stationary Phase Retention in the Cross-Axis Countercurrent Chromatography. The cross-axis countercurrent chromatography ( $X$-axis CCC) was designed and fabricated in our laboratory. The $X$-axis CCC apparatus is displayed as Figure 2, which includes the six separation columns at the total column capacity of $71 \mathrm{~mL}$. Each column was tightly coiled by the polytetrafluoroethylene (PTFE) tube with $2.0 \mathrm{~mm}$ inner diameter. In view of the best experimental partition efficiencies of ATPS in $X$-axis CCC [32], the measurement of stationary phase retention of ATPS in our $X$-axis CCC was designed as follows: the bottom phase was selected as mobile phase, $X$-axis CCC revolution direction was counterclockwise, the elution mode was from head to tail and from inward to outward, and the flow rate was $0.5 \mathrm{~mL} / \mathrm{min}$. The specific operations for the stationary phase retention measurement were the same as Shinomiya's methods [33].

\section{Results and Discussion}

3.1. The Effect of TLL on ATPS Density, Viscosity, and Interfacial Tension. The effect of TLL on the density can be seen in Figure 3. It has been shown that the densities of both top and bottom phases are increasing as the TLL becomes longer. Compared with the influence of TLL on the density of the top phase, the variation in density of the bottom phase is very significant. This is because the influence of the composition of salt on the density is larger than the influence of the composition of PEG. Furthermore, the bottom is rich in salt and the top phase is rich in PEG. As a result, the density difference between the bottom phase and the top phase increases with the increasing TLL. Moreover, it can be seen that the PEG molecular weight has no obvious effect on the density of the PEG-rich phase of ATPS compared to Figures 3(a) and 3(b) or Figures 3(c) and 3(d).

The viscosities of the top and bottom phases as a function of the TLL are shown in Figure 4. Figure 4 shows that the viscosity of the top phase increases slightly with the increasing TLL. Furthermore, there is a marked change in viscosity of PEG4000 ATPS as TLL increasing compared with PEG1000 ATPS. It is because the viscosities of ATPS increase with the increasing molar mass of PEG at the same PEG concentration. Perumalsamy and Murugesan have measured the viscosity of PEG solutions at $25^{\circ} \mathrm{C}$ and observed the same result [34].

Figure 5 shows that the interfacial tension increases with the increasing TLL for all the ATPS. Mishima et al. used the drop volume technique and observed the same trend [35]. The compositions' concentrations play a key role in the solution's interfacial tension. In ATPS, the compositions' concentrations increase with the increase of TLL in both the top phase and the bottom phase. Thus, the interfacial tensions in various ATPS are significantly enhanced with the increasing TLL.

3.2. The Separation Behavior of ATPS. The separation behaviors of four ATPS are shown in Figure 6. As can be seen in Figure 6, to the left of the solid points, the top phase of ATPS is continuous. To the right of the hollow points, the bottom 


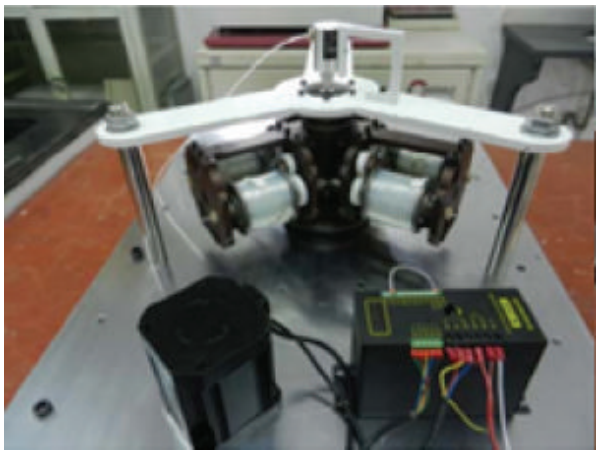

(a)

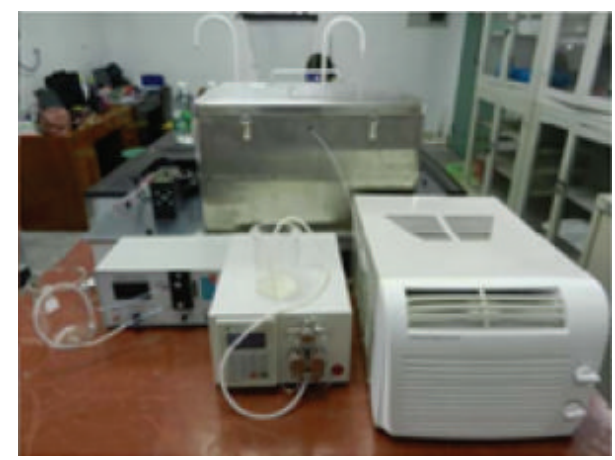

(b)

FIGURE 2: Photographs of our cross-axis countercurrent chromatography without the cover case (a) and the overall appearance (b).

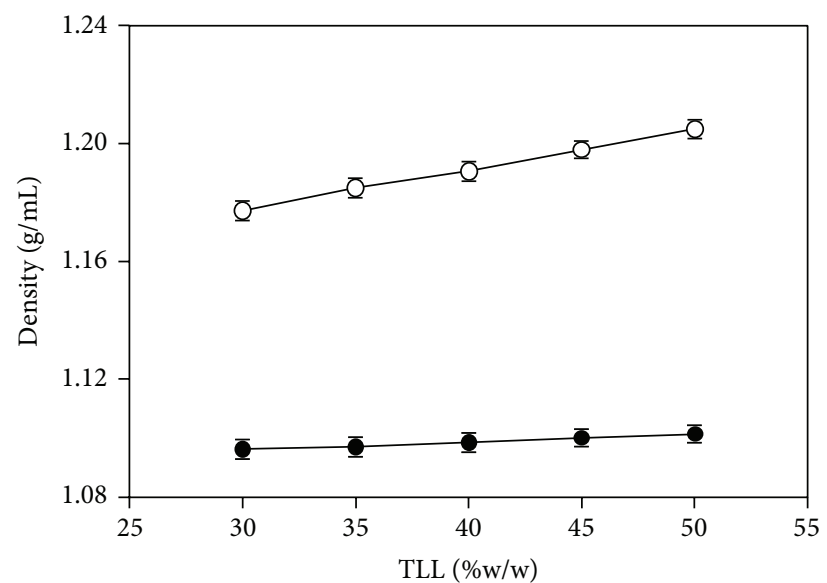

(a)

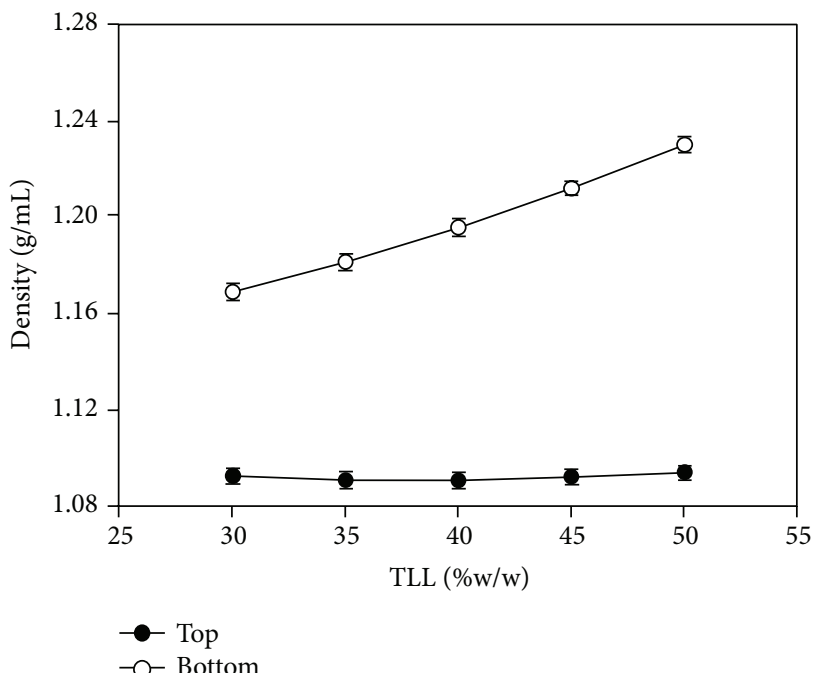

(c)

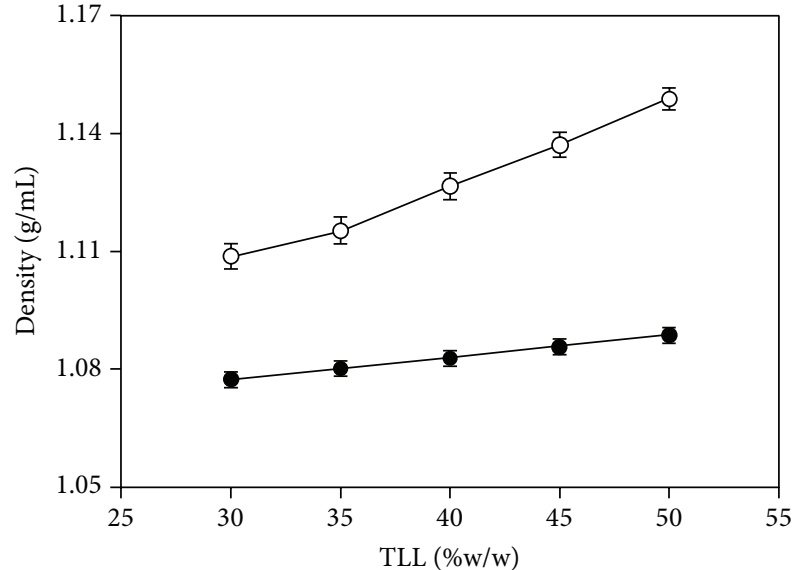

(b)

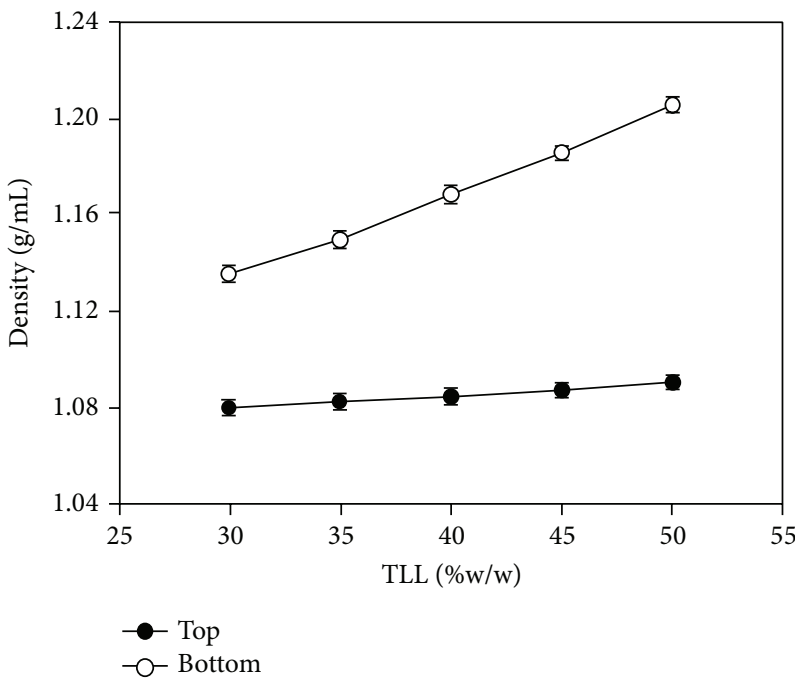

(d)

Figure 3: Densities of the top phase and the bottom phase as a function of TLL at $25^{\circ} \mathrm{C}$. (a) PEG1000/ammonium sulfate ATPS, (b) PEG4000/ammonium sulfate ATPS, (c) PEG1000/sodium citrate ATPS, and (d) PEG4000/sodium citrate ATPS. The error bars represent the standard deviations in triplicate experiments. 


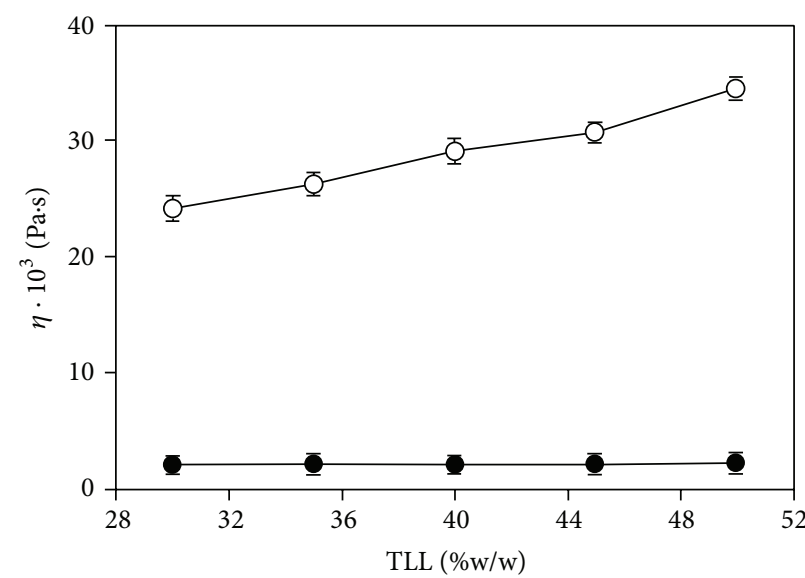

(a)

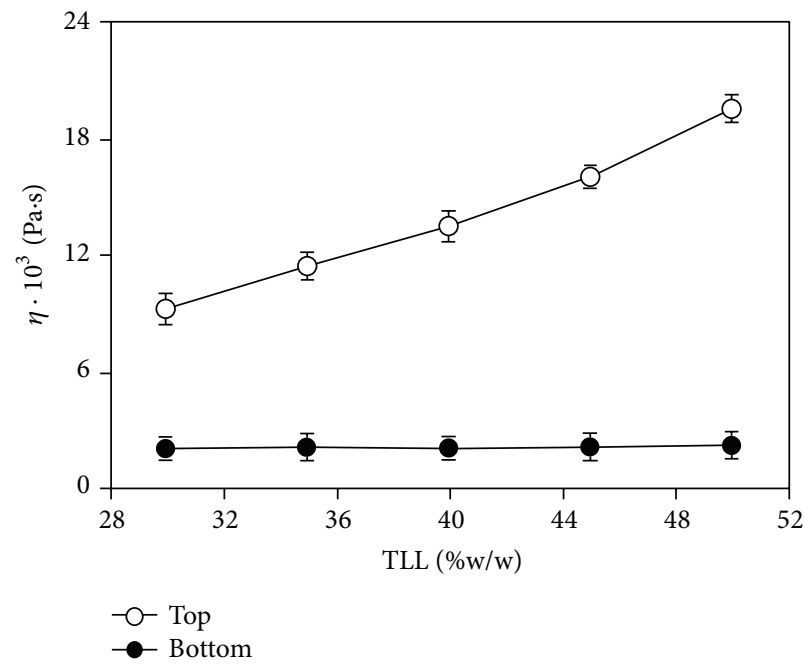

(c)

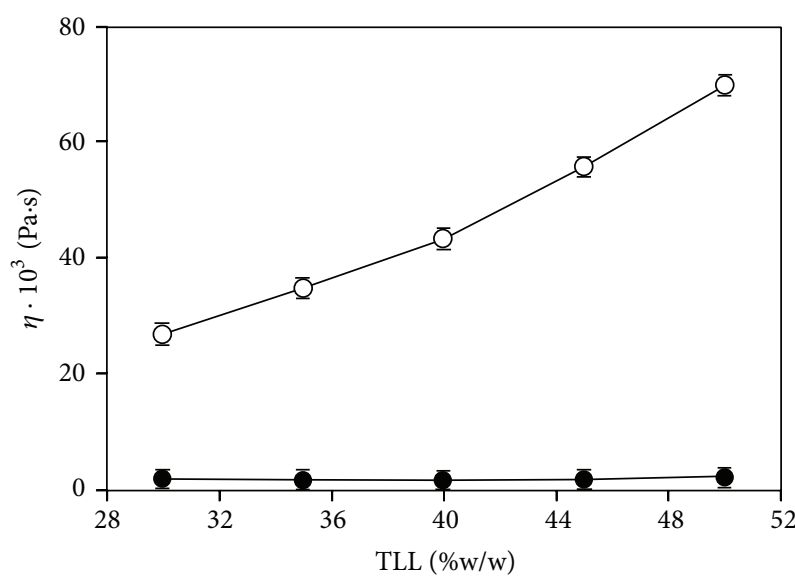

(b)

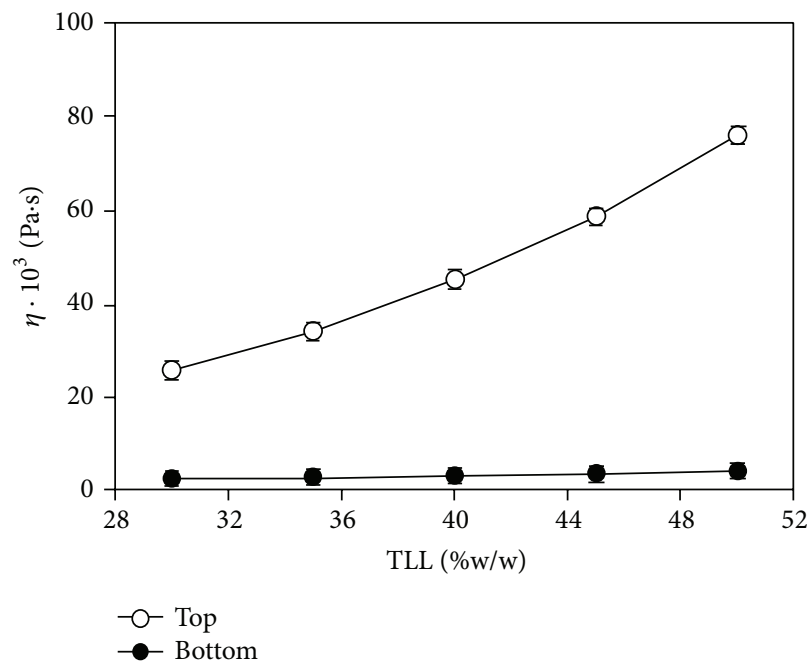

(d)

FIGURE 4: Viscosities of the top phase and the bottom phase as a function of TLL at $25^{\circ} \mathrm{C}$. (a) PEG1000/ammonium sulfate ATPS, (b) PEG4000/ammonium sulfate ATPS, (c) PEG1000/sodium citrate ATPS, and (d) PEG4000/sodium citrate ATPS. The error bars represent the standard deviations in triplicate experiments.

phase of ATPS is continuous. The region which is called phase inversion band between the solid points and hollow points on the same TLL is a range of ambiguity. Both top-continuous phase and bottom-continuous phase can be seen in this region of ATPS. Within this region, the continuity of the phase is affected not only by composition of the mixture, but also by the fluid dynamics. The region of ambiguity becomes large with the increasing TLL and locates nearly at the constant salt concentration line. In Figure 6, the region of bottom-continuous phase is almost 4 times larger than the region of top-continuous phase. Furthermore, the middle points of TLL locate in the bottom-continuous phase. This indicates that the four investigated ATPS of $V_{r}=1$ were operated in the region of bottom-continuous phase.

Figure 7 shows the profile of the phase separation time changes with the increasing top phase ratio $\left(V_{t}=1 /\left(V_{r}+1\right)\right)$. Phase inversion takes place near the point of $V_{t}=0.8\left(V_{r}=\right.$ 4 ), where the continuous phase can change into the dispersed phase, and a sudden change of phase separation behavior is observed. The bottom phase is continuous when the $V_{t}$ is less than 0.8 , and short phase separation times are observed with the smooth variation. The top phase is continuous when the $V_{t}$ is more than 0.8 , and the dramatic increase of phase separation time with the increasing $V_{t}$ is observed. It indicates that ATPS would be better to operate in the bottomcontinuous region rather than top-continuous region.

3.3. Correlation Equations of Phase Separation. Since the ATPS of $V_{r}=1$ have been widely applied in the separation of biological products, the phase separation rate of ATPS of $V_{r}=$ 1 has been investigated. The surface tension of water $\left(\sigma_{w}\right)$ can be used in the equation rather than the surface tension of continuous phase $\left(\sigma_{c}\right)$. Hence, (2) can be modified as

$$
\frac{d h}{d t}\left(\frac{\mu_{c}}{\lambda}\right)=a M_{0}^{b}\left(\frac{\gamma}{\sigma_{w}}\right)^{c}(\mathrm{TLL})^{d} .
$$




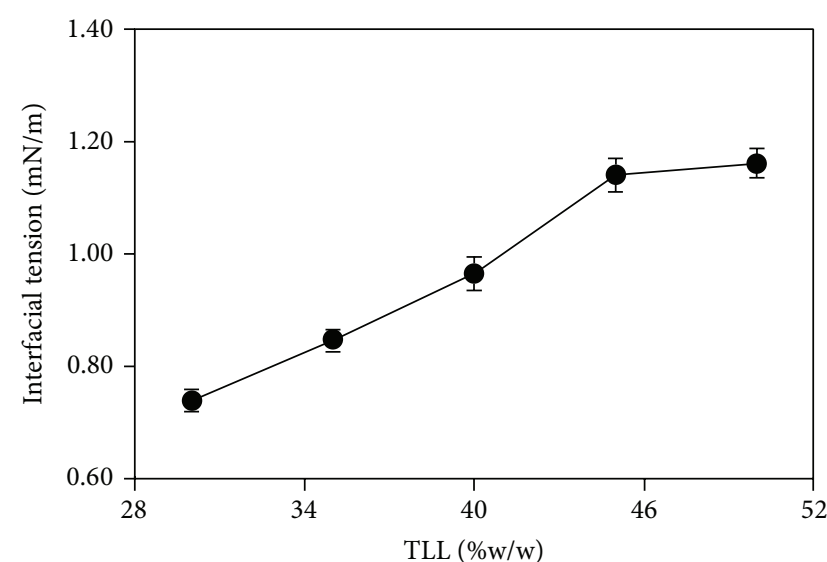

(a)

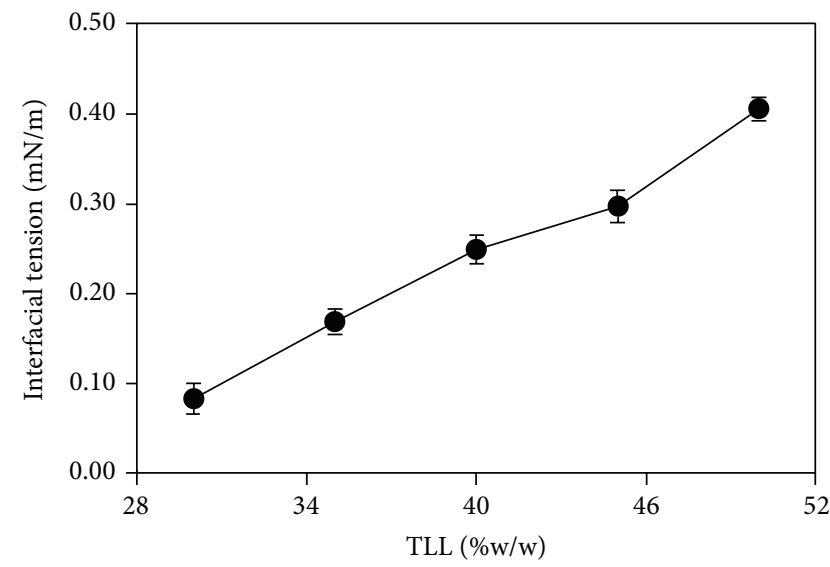

(c)

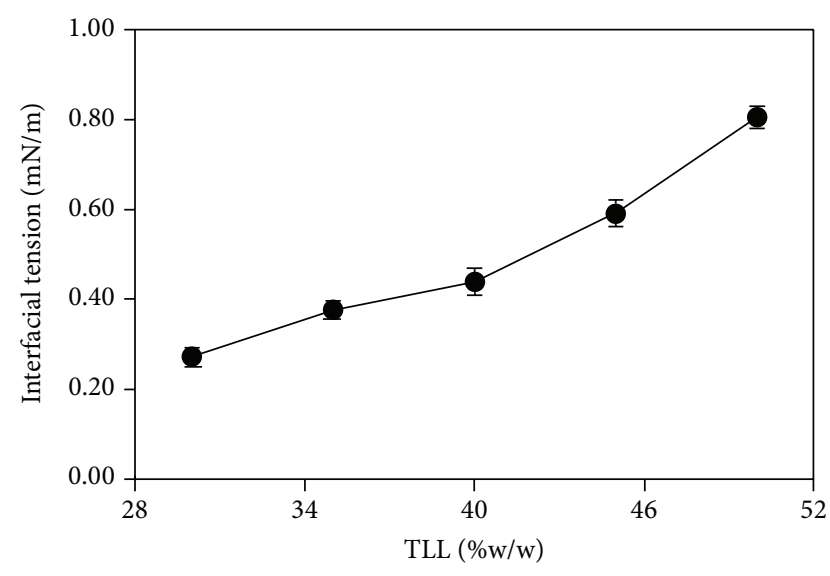

(b)

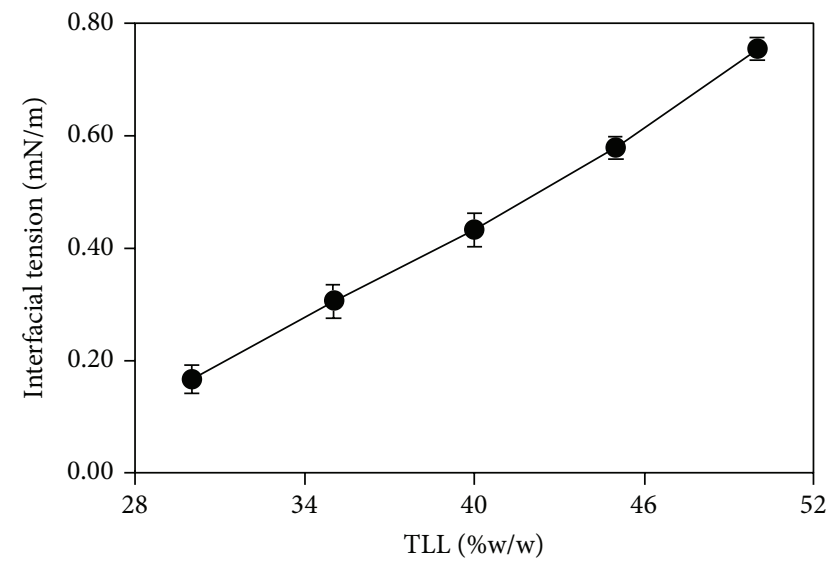

(d)

FIgURE 5: Interfacial tension between the top phase and the bottom phase as a function of TLL at $25^{\circ} \mathrm{C}$. (a) PEG1000/ammonium sulfate ATPS, (b) PEG4000/ammonium sulfate ATPS, (c) PEG1000/sodium citrate ATPS, and (d) PEG4000/sodium citrate ATPS. The error bars represent the standard deviations in triplicate experiments.

The value of the coefficients $(a, b, c, d)$ can be calculated with the experimental values (Tables 1 and 2). This was performed by nonlinear regression using 1stOpt 1.5 software (China). Using these values for the bottom-continuous region, (4) can be written as (5) and (6).

For the bottom-continuous region of PEG/ammonium sulfate ATPS, the correlation equation can be expressed:

$$
\frac{d h}{d t}\left(\frac{\mu_{b}}{\gamma}\right)=3.58 \times 10^{-3} M_{0}^{0.0080}\left(\frac{\gamma}{\sigma_{w}}\right)^{-1.8688}(\mathrm{TLL})^{0.7650}
$$

For the bottom-continuous region of PEG/sodium citrate ATPS, the correlation equation can be expressed:

$$
\frac{d h}{d t}\left(\frac{\mu_{b}}{\gamma}\right)=1.05 \times 10^{-6} M_{0}^{0.1735}\left(\frac{\gamma}{\sigma_{w}}\right)^{-1.5403}(\mathrm{TLL})^{2.5750} .
$$

The $R^{2}$ (correlation index) of the two equations is 0.960 for PEG/ammonium sulfate ATPS and 0.971 for PEG/sodium citrate ATPS, respectively. Equation (5) was used to predict the phase separation rate of the bottom-continuous region of PEG/ammonium sulfate ATPS of $V_{r}=1$ with an average absolute relative deviation (AARD) value of 7.05\%. Equation (6) was used to predict the phase separation rate of the bottom-continuous region of PEG/sodium citrate ATPS of $V_{r}=1$ with an AARD value of $6.81 \%$. The values of the coefficient indicate the difference of the phase separation rates between the two ATPS. The two equations show that the TLL has a greater effect on the PEG/sodium citrate ATPS than the PEG/ammonium sulfate ATPS. The $M_{0}$ has a similar effect on the phase separation rates of both ATPS.

Both (5) and (6) have predicted the phase separation rates of ATPS with $V_{r}=1$. For the batch separation, the results of prediction can provide a reference since the stable ATPS with $V_{r}=1$ are widely used. For the continuous separation, such as HSCCC, the phase separation rate is the most important factor for the choice of solvent systems [36]. Hence, it is necessary to investigate the relationship between phase separation rate and physical properties of ATPS. In this paper, as phase diagrams show in Figure 6, the bottomcontinuous regions of the four investigated ATPS are fit for continuous separation due to the fast phase separation rates and weak $V_{r}$ dependence. 


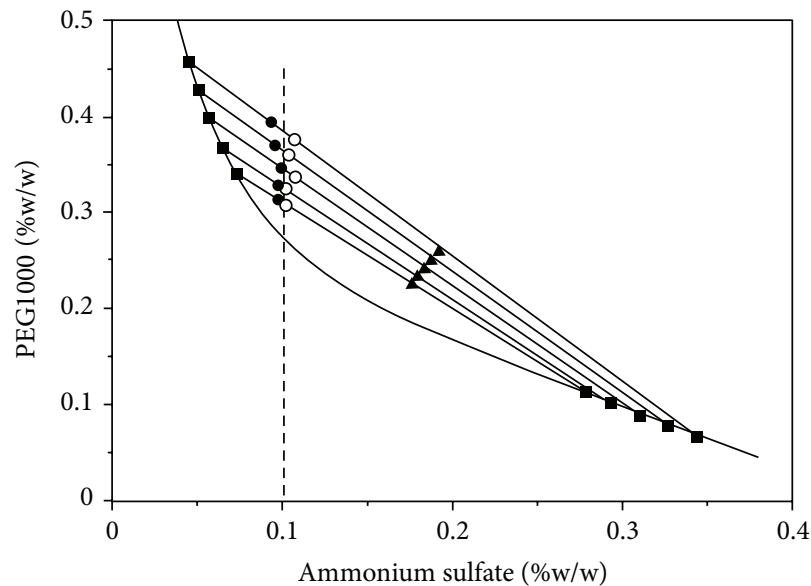

(a)

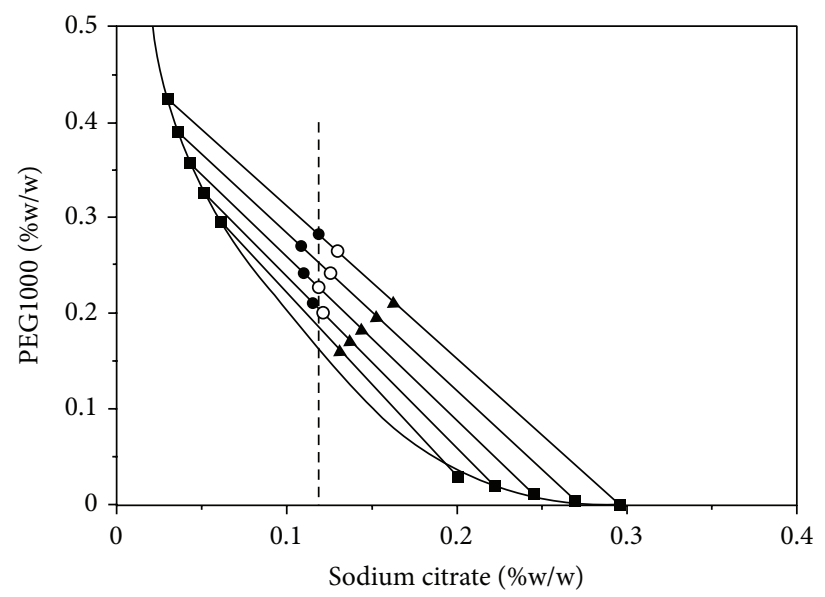

(c)

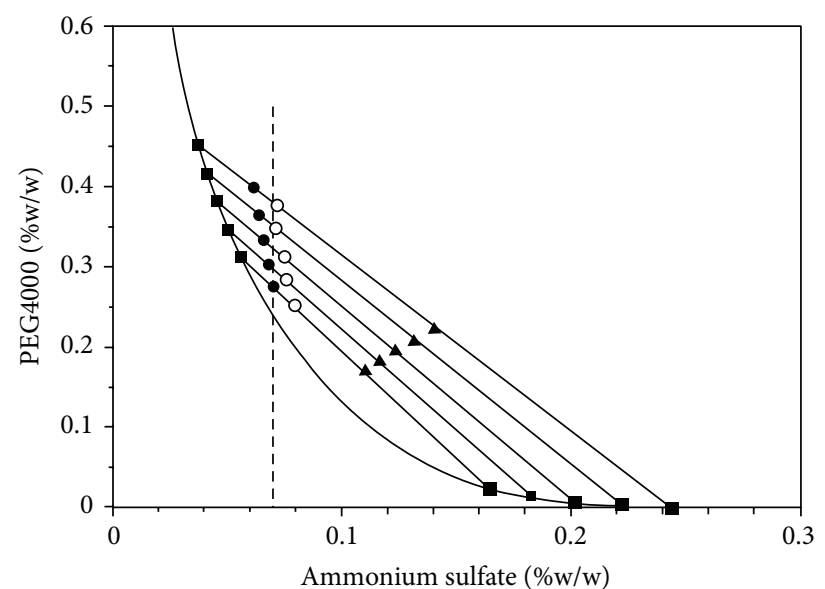

(b)

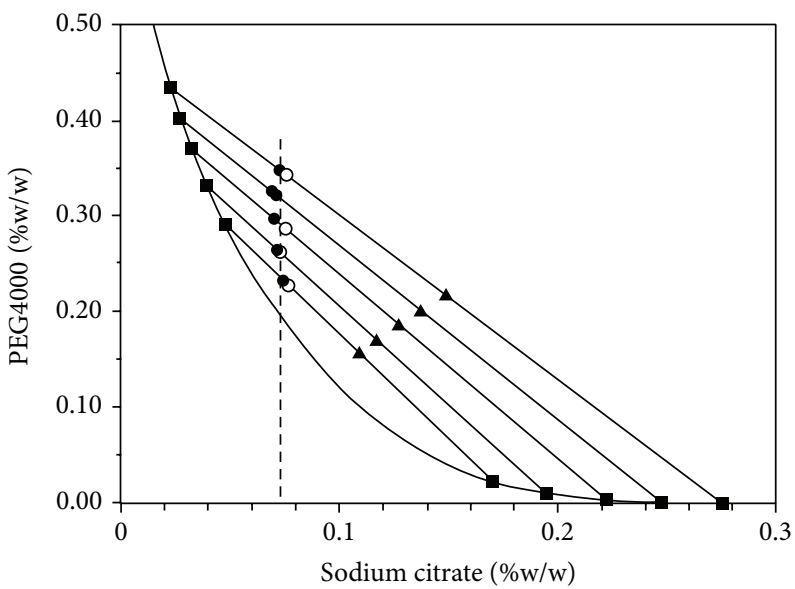

(d)

Figure 6: Experimental equilibrium data obtained for the ATPS at $25^{\circ} \mathrm{C}$, phase inversion band between the points at the same TLL. (a) PEG1000/ammonium sulfate ATPS, (b) PEG4000/ammonium sulfate ATPS, (c) PEG1000/sodium citrate ATPS, and (d) PEG4000/sodium citrate ATPS.

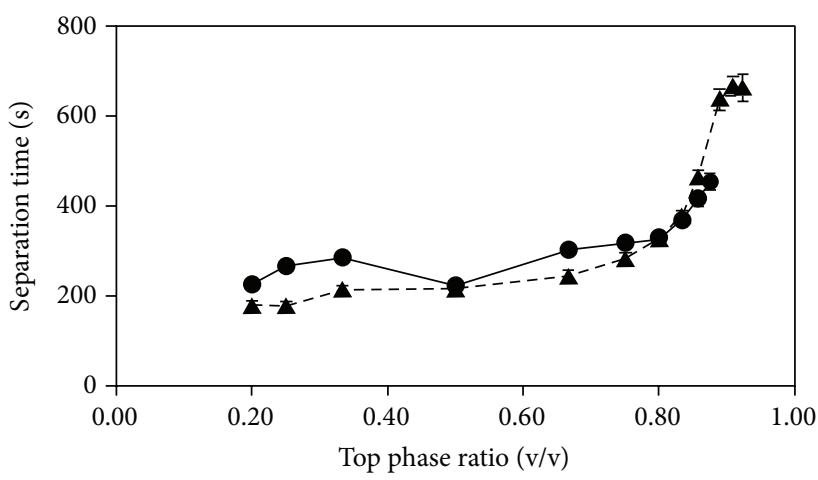

$-\mathbf{\Delta}-\mathrm{TLL}=50$

- $\mathrm{TLL}=40$

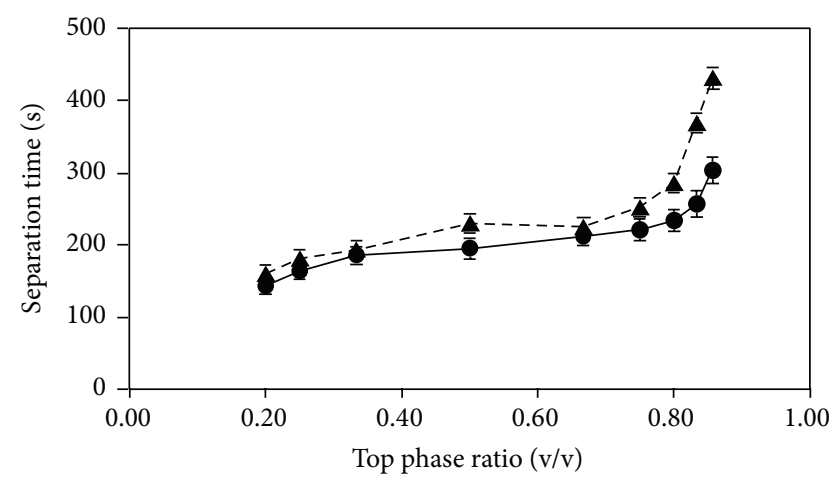

$-\mathbf{\Delta}-\mathrm{TLL}=50$

$\mathrm{TLL}=40$

(a)

(b)

Figure 7: Effect of the change in percentage top phase $\left(V_{t}=1 /\left(V_{r}+1\right)\right)$ on phase separation time for the ATPS at $25^{\circ} \mathrm{C}$. (a) PEG4000/ammonium sulfate ATPS and (b) PEG4000/sodium citrate ATPS. The error bars represent the standard deviations in triplicate experiments. 
TABLE 1: Experimental properties parameters of PEG1000/ammonium sulfate ATPS and PEG4000/ammonium sulfate ATPS at $25^{\circ} \mathrm{C}$.

\begin{tabular}{lcccccc}
\hline & $\begin{array}{c}\mathrm{TLL} \\
{[\% \mathrm{w} / \mathrm{w}]}\end{array}$ & $\begin{array}{c}\Delta t \\
{[\mathrm{~s}]}\end{array}$ & $\begin{array}{c}\sigma_{w} \\
{[\mathrm{mN} / \mathrm{m}]}\end{array}$ & $\begin{array}{c}\gamma \\
{[\mathrm{mN} / \mathrm{m}]}\end{array}$ & $\begin{array}{c}\Delta \mu \\
{[\mathrm{mPa} \cdot \mathrm{s}]}\end{array}$ & $\begin{array}{c}\mu_{b} \\
{[\mathrm{mPa} \cdot \mathrm{s}]}\end{array}$ \\
\hline \multirow{3}{*}{ PEG1000/ } & 30 & 90.38 & 71.97 & 0.739 & 22.130 & 2.120 \\
ammonium & 35 & 93.46 & 71.97 & 0.846 & 24.158 & 2.193 \\
sulfate & 40 & 97.52 & 71.97 & 0.965 & 27.053 & 2.148 \\
& 45 & 96.44 & 71.97 & 1.140 & 28.595 & 2.205 \\
& 50 & 101.04 & 71.97 & 1.161 & 32.305 & 2.295 \\
\hline & 30 & 280.62 & 71.97 & 0.272 & 25.010 & 1.990 \\
PEG4000/ & 35 & 247.02 & 71.97 & 0.376 & 33.085 & 1.865 \\
ammonium & 40 & 218.88 & 71.97 & 0.439 & 41.625 & 1.775 \\
sulfate & 45 & 188.00 & 71.97 & 0.592 & 54.005 & 1.895 \\
& 50 & 164.10 & 71.97 & 0.805 & 67.790 & 2.210 \\
\hline
\end{tabular}

TABLE 2: Experimental properties parameters of PEG1000/sodium citrate ATPS and PEG4000/sodium citrate ATPS at $25^{\circ} \mathrm{C}$.

\begin{tabular}{lcccccc}
\hline & $\begin{array}{c}\text { TLL } \\
{[\% \mathrm{w} / \mathrm{w}]}\end{array}$ & $\begin{array}{c}\Delta t \\
{[\mathrm{~s}]}\end{array}$ & $\begin{array}{c}\sigma_{w} \\
{[\mathrm{mN} / \mathrm{m}]}\end{array}$ & $\begin{array}{c}\gamma \\
{[\mathrm{mN} / \mathrm{m}]}\end{array}$ & $\begin{array}{c}\Delta \mu \\
{[\mathrm{mPa} \cdot \mathrm{s}]}\end{array}$ & $\begin{array}{c}\mu_{b} \\
{[\mathrm{mPa} \cdot \mathrm{s}]}\end{array}$ \\
\hline \multirow{3}{*}{ PEG1000/ } & 30 & 156.18 & 71.97 & 0.083 & 5.650 & 3.650 \\
sodium & 40 & 133.48 & 71.97 & 0.169 & 7.780 & 3.720 \\
citrate & 45 & 111.98 & 71.97 & 0.297 & 11.68 & 4.420 \\
& 50 & 111.32 & 71.97 & 0.405 & 14.365 & 5.235 \\
\hline & 30 & 279.93 & 71.97 & 0.167 & 23.165 & 2.435 \\
PEG4000/ & 35 & 247.85 & 71.97 & 0.305 & 31.185 & 2.715 \\
sodium & 40 & 244.29 & 71.97 & 0.433 & 42.110 & 2.940 \\
citrate & 45 & 267.05 & 71.97 & 0.579 & 55.145 & 3.355 \\
& 50 & 266.48 & 71.97 & 0.755 & 71.985 & 4.015 \\
\hline
\end{tabular}

3.4. Stationary Phase Retention of PEG1000/Sodium Citrate ATPS in the X-Axis CCC. PEG1000/sodium citrate ATPS were selected to investigate the positive effect of fast phase separation rate on the stationary phase retention in the crossaxis countercurrent chromatography. According to the above correlation equation (6), the shorter TLL of PEG1000/sodium citrate ATPS could obtain the faster phase separation rate of the two phases. Thus, PEG1000/sodium citrate ATPS with TLL $=30(16.54 \mathrm{w} / \mathrm{w} \%$ PEG1000 and $13.96 \mathrm{w} / \mathrm{w} \%$ sodium citrate) was determined as the investigated ATPS for the measurement of the stationary phase retention in our $X$-axis CCC at various rotation rates. Figure 8 displays that the effect of the $X$-axis CCC revolution speed on the stationary phase retention of PEG1000/sodium citrate ATPS with TLL $=30$. It can be seen that the maximum value of stationary phase retention was $54.83 \%$ at $800 \mathrm{rpm}$, which was slightly higher than the reported stationary phase retention of ATPS in other $X$-axis CCCs [32]. It indicated that the phase separation rate of ATPS played an important role in the stationary phase retention in HSCCC.

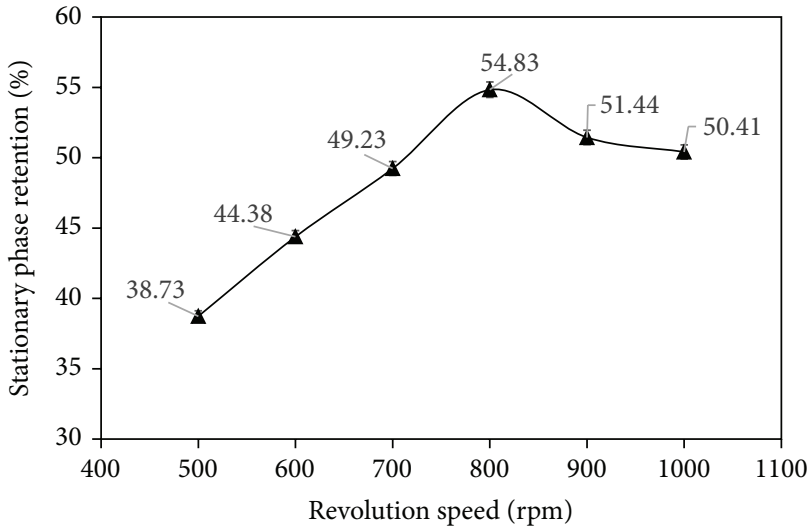

FIGURE 8: Effect of revolution speed on stationary phase retention of PEG1000/sodium citrate in the $X$-axis CCC. The error bars represent the standard deviations in triplicate experiments.

\section{Conclusion}

The physical properties of PEG/ammonium sulfate and PEG/sodium citrate ATPS had been studied. The density of bottom phase is larger than that of top phase and increases with increasing TLL. The viscosity of the top phase is 3-35 times larger than the bottom phase and increases with the increasing TLL. The interfacial tension between the phases increases with the increasing TLL.

The bottom phase is continuous at high $V_{r}$ value, and fast separation rate has been observed as well as the smooth variation. The top phase is continuous at low $V_{r}$ value, and the dramatic increase of phase separation time with the increasing $V_{r}$ value was observed. There is a phase inversion band between the top-continuous region and bottom-continuous region. The phase inversion band is located at the constant salt concentration line in the phase diagram. Within this region, the continuity of the phase is affected not only by the composition of the ATPS, but also by the fluid dynamics.

The phase separation rate was correlated using a modified correlation equation and the coefficients were found. It showed that the $R^{2}$ of the two equations were 0.960 for PEG/ ammonium sulfate ATPS and 0.971 for PEG/sodium citrate ATPS, respectively. The correlation equations gave good results for the bottom-continuous ATPS with $V_{r}=1$. The results may be useful for the choice of optimal conditions of ATPS no matter in batch separation or continuous separation process. The correlation equations were applied to estimate the fast phase separation rate of PEG1000/sodium citrate ATPS and further to measure the stationary phase retention of PEG/sodium citrate ATPS in our $X$-axis CCC, in which the higher stationary phase retention was obtained at $800 \mathrm{rpm}$.

\section{Nomenclature}

$R^{2}$ : Correlation index,

$$
R^{2}=\frac{\left(\sum_{i=1}^{n} x_{i} y_{i}-\sum_{i=1}^{n} x_{i} \sum_{i=1}^{n} y_{i} / n\right)^{2}}{\left[\sum_{i=1}^{n} x_{i}^{2}-\left(\sum_{i=1}^{n} x_{i}\right)^{2} / n\right]\left[\sum_{i=1}^{n} y_{i}^{2}-\left(\sum_{i=1}^{n} y_{i}\right)^{2} / n\right]},
$$


where $x_{i}, y_{i}$, and $n$ are the experimental, calculated, and number of data points, respectively

AARD: Average absolute relative deviation,

$$
\mathrm{AARD}=\left(\sum_{i=1}^{n}\left|\frac{y_{\mathrm{exp}, i}-y_{\mathrm{cal}, i}}{y_{\mathrm{exp}, i}} \times 100 \%\right|\right) \times\left(\frac{1}{n}\right),
$$

where $y_{\mathrm{exp}, i}, y_{\mathrm{cal}, i}$, and $n$ are the experimental, calculated, and number of data points, respectively

$\Delta h$ : Height of the interphase

$\Delta t$ : Time of phase separation

$V_{\text {tp }}$ : Volume of top phase

$V_{\mathrm{bp}}$ : Volume of bottom phase

$M_{0}$ : Morton number.

\section{Greek Letters}

$\Delta \rho$ : Density difference between the top and bottom phases

$\rho_{c}$ : Density of the continuous phase

$\gamma$ : Interfacial tension between the top and bottom phases

$\sigma_{w}$ : Surface tension of the air-water interface at $25^{\circ} \mathrm{C}$

$\sigma_{c}$ : Surface tension between air-continuous phase interface

$\mu_{c}$ : Viscosity of the continuous phase

$\mu_{d}$ : Viscosity of the dispersed phase

$\mu_{b}$ : Viscosity of the bottom-continuous phase

$\Delta \mu$ : Viscosity difference between the phases.

\section{Conflict of Interests}

The authors declare that there is no conflict of interests regarding the publication of this paper.

\section{Acknowledgments}

This work is supported by the National Natural Science Foundation of China (no. 21476135), Outstanding Young Teachers Training Program in Guangdong Higher Education Institutions (no. Yq2013076), Science and Technology Planning Project of Guangdong Province, China (no. 2012B060400006), and Education Department Projects of Guangdong Province, China (nos. 2012KJCX0052 and 92038030337).

\section{References}

[1] J. Benavides and M. Rito-Palomares, "Practical experiences from the development of aqueous two-phase processes for the recovery of high value biological products," Journal of Chemical Technology and Biotechnology, vol. 83, no. 2, pp. 133-142, 2008.
[2] J. Benavides, O. Aguilar, B. H. Lapizco-Encinas, and M. RitoPalomares, "Extraction and purification of bioproducts and nanoparticles using aqueous two-phase systems strategies," Chemical Engineering and Technology, vol. 31, no. 6, pp. 838$845,2008$.

[3] D. F. C. Silva, A. M. Azevedo, P. Fernandes, V. Chu, J. P. Conde, and M. R. Aires-Barros, "Design of a microfluidic platform for monoclonal antibody extraction using an aqueous two-phase system," Journal of Chromatography A, vol. 1249, pp. 1-7, 2012.

[4] S. L. Mistry, A. Kaul, J. C. Merchuk, and J. A. Asenjo, "Mathematical modelling and computer simulation of aqueous twophase continuous protein extraction," Journal of Chromatography A, vol. 741, no. 2, pp. 151-163, 1996.

[5] P. A. Albertsson, Partition of Cell Particles and Macromolecules, Wiley-Interscience, New York, NY, USA, 1986.

[6] B. Y. Zaslasvsky, Aqueous Two-Phase Partitioning: Physical Chemistry and Bioanalytical Applications, Marcel Dekker, New York, NY, USA, 1995.

[7] M. Rito-Palomares, "Practical application of aqueous two-phase partition to process development for the recovery of biological products," Journal of Chromatography B: Analytical Technologies in the Biomedical and Life Sciences, vol. 807, no. 1, pp. 3-11, 2004.

[8] E. Huenupi, A. Gomez, B. A. Andrews, and J. A. Asenjo, "Optimization and design considerations of two-phase continuous protein separation," Journal of Chemical Technology \& Biotechnology, vol. 74, no. 3, pp. 256-263, 1999.

[9] S. Saravanan, J. R. Rao, T. Murugesan, B. U. Nair, and T. Ramasami, "Recovery of value-added globular proteins from tannery wastewaters using PEG-salt aqueous two-phase systems," Journal of Chemical Technology \& Biotechnology, vol. 81, no. 11, pp. 1814-1819, 2006.

[10] P. A. J. Rosa, I. F. Ferreira, A. M. Azevedo, and M. R. AiresBarros, "Aqueous two-phase systems: a viable platform in the manufacturing of biopharmaceuticals," Journal of Chromatography A, vol. 1217, no. 16, pp. 2296-2305, 2010.

[11] K. H. Kroner, H. Schütte, W. Stach, and M.-R. Kula, "Scaleup of formate dehydrogenase by partition," Journal of Chemical Technology and Biotechnology, vol. 32, no. 1, pp. 130-137, 1982.

[12] K. Selber, F. Tjerneld, A. Collén et al., "Large-scale separation and production of engineered proteins, designed for facilitated recovery in detergent-based aqueous two-phase extraction systems," Process Biochemistry, vol. 39, no. 7, pp. 889-896, 2004.

[13] Y. Zeng, G. Liu, Y. Ma, X. Y. Chen, and Y. Ito, "Organic high ionic strength aqueous two-phase solvent system series for separation of ultra-polar compounds by spiral high-speed counter-current chromatography," Journal of Chromatography A, vol. 1218, no. 48, pp. 8715-8717, 2011.

[14] M. H. Salamanca, J. C. Merchuk, B. A. Andrews, and J. A. Asenjo, "On the kinetics of phase separation in aqueous twophase systems," Journal of Chromatography B: Biomedical Applications, vol. 711, no. 1-2, pp. 319-329, 1998.

[15] A. V. Narayan, M. C. Madhusudhan, and K. S. M. S. Raghavarao, "Demixing kinetics of phase systems employed for liquid-liquid extraction and correlation with system properties," Food and Bioproducts Processing, vol. 89, no. 4, pp. 251-256, 2011.

[16] K. Shinomiya, H. Kobayashi, N. Inokuchi et al., "New smallscale cross-axis coil planet centrifuge. Partition efficiency and application to purification of bullfrog ribonuclease," Journal of Chromatography A, vol. 1151, no. 1-2, pp. 91-98, 2007.

[17] N. Mekaoui, K. Faure, and A. Berthod, "Advances in countercurrent chromatography for protein separations," Bioanalysis, vol. 4, no. 7, pp. 833-844, 2012. 
[18] K. Shinomiya, H. Kobayashi, N. Inokuchi, K. Nakagomi, and Y. Ito, "Partition efficiency of high-pitch locular multilayer coil for countercurrent chromatographic separation of proteins using small-scale cross-axis coil planet centrifuge and application to purification of various collagenases with aqueous-aqueous polymer phase systems," Journal of Liquid Chromatography \& Related Technologies, vol. 34, no. 3, pp. 182-194, 2011.

[19] G. Tubio, G. A. Picó, and B. B. Nerli, "Extraction of trypsin from bovine pancreas by applying polyethyleneglycol/sodium citrate aqueous two-phase systems," Journal of Chromatography B: Analytical Technologies in the Biomedical and Life Sciences, vol. 877, no. 3, pp. 115-120, 2009.

[20] R. L. Pérez, D. B. Loureiro, B. B. Nerli, and G. Tubio, "Optimization of pancreatic trypsin extraction in PEG/citrate aqueous two-phase systems," Protein Expression and Purificatio, vol. 106, pp. 66-71, 2015.

[21] M. Perumalsamy and T. Murugesan, "Prediction of liquidliquid equilibria for PEG 2000-sodium citrate based aqueous two-phase systems," Fluid Phase Equilibria, vol. 244, no. 1, pp. 52-61, 2006.

[22] Y. Liu, Y. Q. Feng, and Y. J. Zhao, "Liquid-liquid equilibrium of various aqueous two-phase systems: experiment and correlation," Journal of Chemical \& Engineering Data, vol. 58, no. 10, pp. 2775-2784, 2013.

[23] M. C. Wikinson, "Extended use of, and comments on, the drop-weight (drop-volume) technique for the determination of surface and interfacial tensions," Journal of Colloid and Interface Science, vol. 40, pp. 14-26, 1972.

[24] C. Jho and R. Burke, "Drop weight technique for the measurement of dynamic surface tension," Journal of Colloid and Interface Science, vol. 95, no. 1, pp. 61-71, 1983.

[25] J. A. Asenjo, S. L. Mistry, B. A. Andrews, and J. C. Merchuk, "Phase separation rates of aqueous two-phase systems: correlation with system properties," Biotechnology and Bioengineering, vol. 79, no. 2, pp. 217-223, 2002.

[26] E. Barnea and J. Mizrahi, "Separation mechanism of liquidliquid dispersions in a deep-layer gravity settler: part IVcontinuous settler characteristics," Transactions of the Institution of Chemical Engineers, vol. 56, pp. 83-92, 1975.

[27] J. Golob and R. Modic, "Coalescence of liquid/liquid dispersions in gravity settlers," Transactions of IChemE, vol. 55, pp. 207-211, 1977.

[28] A. Kaul, R. A. M. Pereira, J. A. Asenjo, and J. C. Merchuk, "Kinetics of phase separation for polyethylene glycol-phosphate two-phase systems," Biotechnology and Bioengineering, vol. 48, no. 3, pp. 246-256, 1995.

[29] M. T. Zafarani-Moattar, R. Sadeghi, and A. A. Hamidi, "Liquidliquid equilibria of an aqueous two-phase system containing polyethylene glycol and sodium citrate: experiment and correlation," Fluid Phase Equilibria, vol. 219, no. 2, pp. 149-155, 2004.

[30] V. H. Nagaraja and R. Lyyaswami, "Phase demixing studies in aqueous two-phase system with polyethylene glycol (PEG) and sodium citrate," Chemical Engineering Communications, vol. 200, no. 10, pp. 1293-1308, 2013.

[31] T. Murugesan and I. Regupathi, "Prediction of continuous phase axial mixing in rotating disc contactors," Journal of Chemical Engineering of Japan, vol. 37, no. 10, pp. 1293-1302, 2004.

[32] K. Shinomiya, K. Yanagidaira, and Y. Ito, "New small-scale cross-axis coil planet centrifuge: the design of the apparatus and its application to counter-current chromatographic separation of proteins with aqueous-aqueous polymer phase systems," Journal of Chromatography A, vol. 1104, no. 1-2, pp. 245-255, 2006.

[33] Y. Shibusawa, Y. Eriguchi, and Y. Ito, "Purification of lactic acid dehydrogenase from bovine heart crude extract by counter-current chromatography," Journal of Chromatography B: Biomedical Applications, vol. 696, no. 1, pp. 25-31, 1997.

[34] M. Perumalsamy and T. Murugesan, "Phase compositions, molar mass, and temperature effect on densities, viscosities, and liquid-liquid equilibrium of polyethylene glycol and salt-based aqueous two-phase systems," Journal of Chemical \& Engineering Data, vol. 54, no. 4, pp. 1359-1366, 2009.

[35] K. Mishima, K. Matsuyama, M. Ezawa, Y. Taruta, S. Takarabe, and M. Nagatani, "Interfacial tension of aqueous two-phase systems containing poly(ethylene glycol) and dipotassium hydrogenphosphate," Journal of Chromatography B: Biomedical Applications, vol. 711, no. 1-2, pp. 313-318, 1998.

[36] Y. Ito, "Golden rules and pitfalls in selecting optimum conditions for high-speed counter-current chromatography," Journal of Chromatography A, vol. 1065, no. 2, pp. 145-168, 2005. 

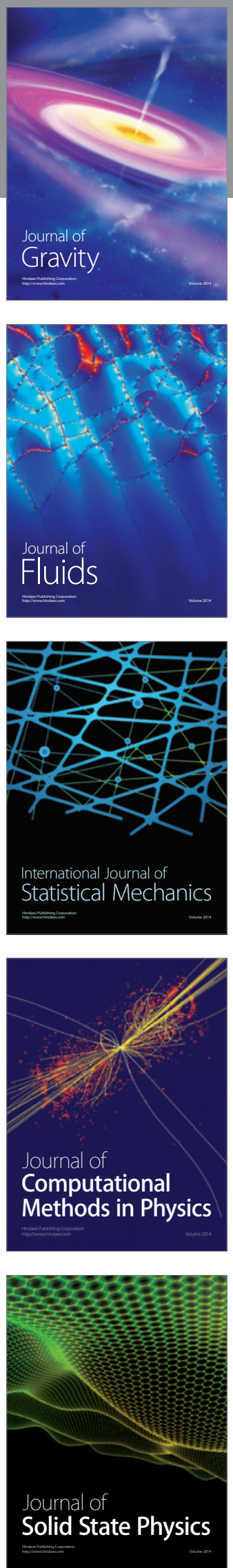

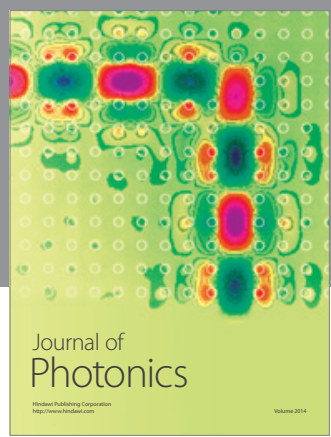

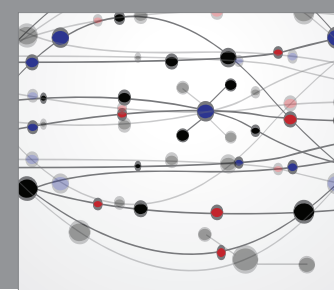

The Scientific World Journal

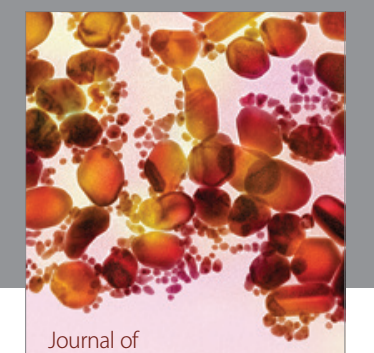

Soft Matter
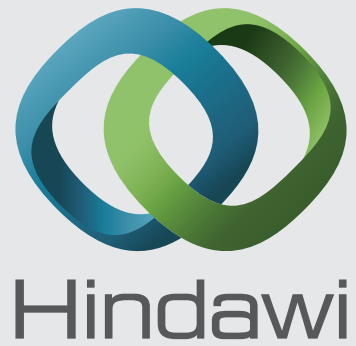

Submit your manuscripts at

http://www.hindawi.com
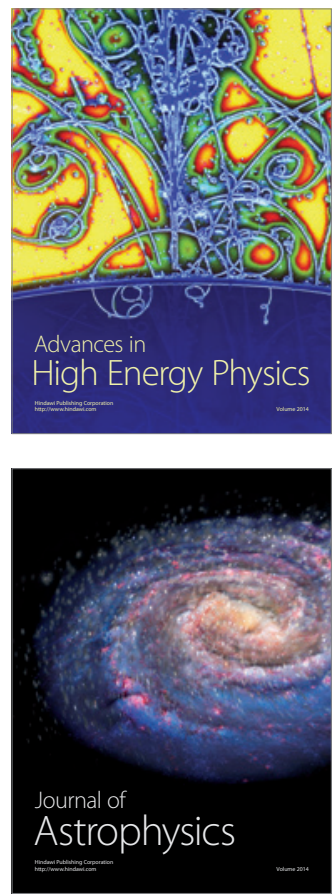
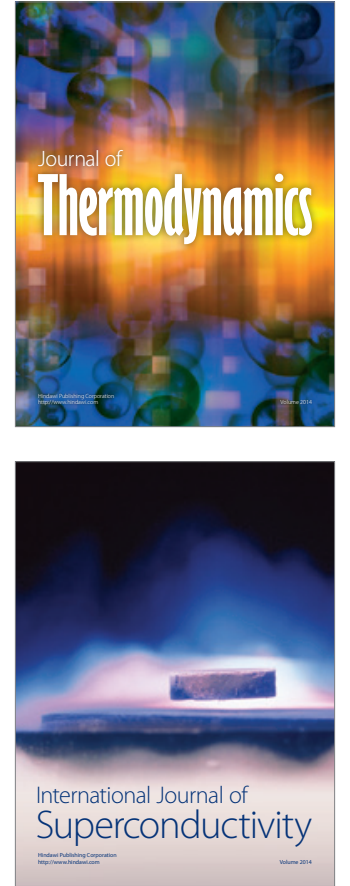
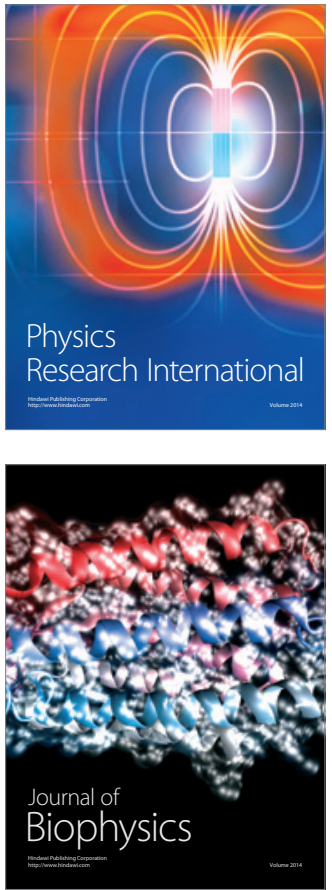
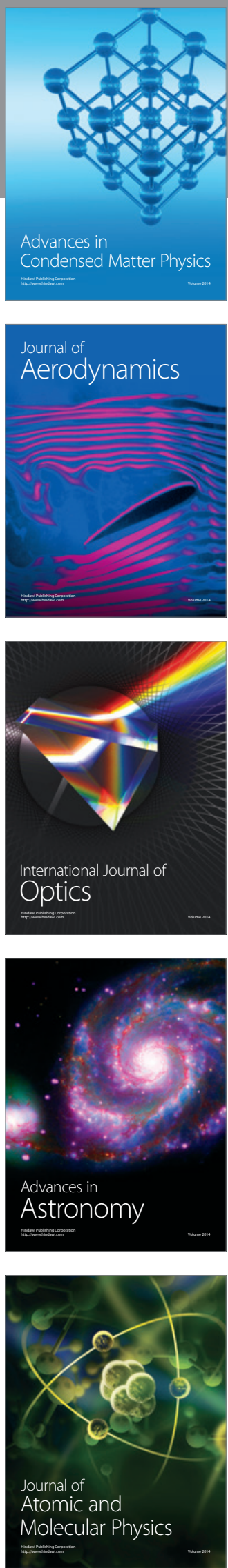